\title{
Influence of collision energy and vibrational excitation on the dynamics for the $\mathrm{H}+\mathrm{HBr} \rightarrow \mathrm{H}_{2}+\mathrm{Br}$ reaction
}

\author{
YANHUA WANG ${ }^{\mathrm{a}}$, MIN PENG $^{\mathrm{b}}$, JIANYING TONG $^{\mathrm{a}}$ and YULIANG WANG ${ }^{\mathrm{c}, *}$ \\ ${ }^{a}$ College of Biology and Environmental Engineering, Zhejiang Shuren University, Hangzhou 310000, China \\ ${ }^{\mathrm{b}}$ Xinjiang Institute of Engineering, Urumqi 830091, China \\ ${ }^{c}$ Department of Basic Sciences, Naval Aeronautical and Astronautical University, Yantai 264001, China \\ e-mail: yarmiay@163.com
}

MS received 15 January 2015; revised 7 June 2015; accepted 9 June 2015

\begin{abstract}
Quasi-classical trajectory (QCT) calculations of $\mathrm{H}+\mathrm{HBr} \rightarrow \mathrm{H}_{2}+\mathrm{Br}$ reaction have been performed on a recently proposed $a b$ initio potential energy surface. The reaction probability and integral cross section are found to be in fairly good agreement with the available quantum mechanical (QM) results on this surface. The behavior of reactivity is well consistent with properties of exothermic reaction. Once the energy of vibrational excited $\mathrm{HBr}$ is larger than the barrier height, the integral cross sections for the reaction diverge at very low collision energies close to the threshold, similarly to capture reaction. In addition, differential cross sections show that scattering of the product $\mathrm{H}_{2}$ shift from backward to forward directions as the collision energy and vibrational quantum number increase. All the theoretical findings are reasonably explained by the properties of the surface, as well as reactive mechanisms.
\end{abstract}

Keywords. Quasi-classical trajectory; reaction dynamics; integral cross section; vibrational excitation.

\section{Introduction}

Collisions between hydrogen atoms and hydrogen halides such as $\mathrm{HF}, \mathrm{HCl}$ and $\mathrm{HBr}$ have been considered to play a very important role in the understanding of chemical kinetics and dynamics from both the theoretical and experimental points of view. Because of the large number of electrons of bromine atom, the calculations of potential energy surface (PES) for $\mathrm{BrH}_{2}$ system are more difficult to perform than that for $\mathrm{FH}_{2}$ and $\mathrm{ClH}_{2}$ systems. Recently, many groups paid attention to this system with experimental and theoretical approaches.

On the experimental side, kinetic isotope effects and reaction rate constants for the exchange and abstraction of $\mathrm{H}+\mathrm{HBr}$ have been the subject of most experiments. ${ }^{1-6}$ The integral cross sections (ICSs) have also been measured at a collision energy of $1.6 \mathrm{eV}$ by coherent anti-Stokes Raman scattering (CARS) spectroscopy, and the results for exchange and abstraction were $11 \pm 2 \AA^{2}$ and $3 \pm 1 \AA^{2}$ respectively. ${ }^{7-9}$ The product quantum state distributions were found to be dominated by both kinematics and PES. Zare and coworkers ${ }^{10}$ measured the product rotational distributions of $\mathrm{H}+\mathrm{HBr} \rightarrow \mathrm{H}_{2}\left(v^{\prime}=2, j^{\prime}\right)+\mathrm{Br}$ at $53 \mathrm{kcal} / \mathrm{mol}$ collision energy employing the photoloc technique and quasiclassical trajectory (QCT) calculations. Recently, they

*For correspondence examined $\mathrm{D}+\mathrm{DBr}(\mathrm{v}=0)$ reaction with an ion imaging method and concluded an almost negligible contribution of the nonadiabatic channel to the reaction, only $1 \%$ of less. ${ }^{11}$

On the theoretical hand, the improvement on the $\mathrm{PESs}^{12-22}$ of $\mathrm{H}_{2} \mathrm{Br}$ motivates the theoretical studies of reaction dynamics for $\mathrm{Br}+\mathrm{H}_{2}$ and its reverse reaction. ${ }^{23-39}$ In fact, the first ab initio PES (denoted as e-LEPS PES) for $\mathrm{H}_{2} \mathrm{Br}$ system was calculated by Lynch et al. ${ }^{15}$ with multi-reference configuration interaction (MRCI) method in 1995. Afterwards, the authors performed quantum scattering calculations on the ground and first electronically excited state potential surfaces to deal with the problem involving an electronically excited product. ${ }^{16}$ Based on this PES, they also calculated the rate constants for $\mathrm{H}+\mathrm{HBr}$ and its isotopic variants. The comparison with available experiments indicated that the representation of the transition state region was not accurate and, especially, the barrier height should be lower. ${ }^{7-9}$ Aiming to solve this question, Kurosaki and Takayanagi constructed a series of global PESs (hereafter denoted as MB $1,{ }^{17}$ $\mathrm{MB} 2^{18}$ and $\mathrm{MB} 3^{19}$ ) of the lowest three doublet states $\left(1^{2} \mathrm{~A}^{\prime}, 2^{2} \mathrm{~A}^{\prime}\right.$, and $\left.1^{2} \mathrm{~A}^{\prime \prime}\right)$ for $\mathrm{Br}+\mathrm{H}_{2}$, using MRCI and complete active space self-consistent field (CASSCF) methods using aug-cc-pVTZ/pVQZ basis sets. The barrier heights for both the exchange and abstraction are smaller than those on e-LEPS potential, leading to a 
relatively better agreement with experiment. Recently, Xie's group constructed a new PES (denoted as JXX1) for the $\mathrm{BrH}_{2}$ system based on the MRCI method including the Davidson's correction, and the agreement of rate constants obtained by time-dependent quantum mechanical (TDQM) calculations with experiments is much improved. ${ }^{20}$ However, the JXX1 PES is nonpublic and used privately. Although there exist some other PESs, ${ }^{12-14,21}$ the e-LEPS, MB1, MB2 and MB3 potential surfaces represent a systematic series of PES for the $\mathrm{H}_{2} \mathrm{Br}$ system, and were used in a large number of dynamical studies. ${ }^{22-31}$ In recent years, Tang's group $^{22-24}$ has performed QM calculations for the $\mathrm{Br}+\mathrm{H}_{2}$ reaction on these PESs, ${ }^{15,17-19}$ and suggested that the MB3 surface provides more reasonable agreement with the experiments than the other three. ${ }^{15,17,18}$ So we employed the MB3 PES for this work.

Note that most of the previous work was focused on the $\mathrm{Br}+\mathrm{H}_{2}$ reaction, less attention has been paid to the reverse reaction $\mathrm{H}+\mathrm{HBr}$. In spite of some dynamical studies done for the $\mathrm{H}+\mathrm{HBr}$ reaction at the initial ground state level $(v=j=0)$, including $\mathrm{QM}^{25,26,32}$ and $\mathrm{QCT}^{29,33}$ calculations, this reaction has not been satisfactorily understood. The above reports basically deal with the scalar properties such as rate constants and ICS and so forth. However, the reagent rovibrational excitation, product rovibrational state distributions and the scattering angle distributions of products with different quantum numbers have not been reported in these literatures. The complete picture on the dynamics of the reaction requires a consideration of these important factors. As the experimental study with a vibrational excited reagent is rather difficult, it would be important and informative to find the mechanism for the influence of reagent vibration on the exothermic reaction dynamics with a theoretical method. To address these questions and gain more insight into the $\mathrm{H}+\mathrm{HBr}$ reaction, we carried out a detailed QCT study for the $\mathrm{H}+\mathrm{HBr}(v=0-3, j=0) \rightarrow \mathrm{H}_{2}+\mathrm{Br}$ on the MB3 surface in the collision energy range $0-2.0 \mathrm{eV}$. As we will show in this paper, a new finding of the switch between activated and capture-type dynamics has been observed at high reagent vibrational excitation for the $\mathrm{H}+\mathrm{HBr}$ abstraction. This phenomenon is in contrast to the general expectation that the vibrational excitation is not the favorable way of inducing reaction for an exothermic reaction with a low and early barrier. The remarkable reaction mechanism and explaining will return in the discussion of the current results.

The paper is organized as follows: Section 2 describes the computational details and the PES used. We present the results and discussion in Section 3. Concluding remarks are summarized in Section 4.

\section{Computational Details}

QCT method is employed as outlined exhaustibly in previous papers. ${ }^{40-46}$ The reader is referred to these publications for more details. Here, we summarize only the most relevant details for the present work.

The initial conditions of the diatomic molecule were randomly sampled by a Monte Carlo procedure. Calculations were performed for the $\mathrm{H}$-atom abstraction reaction with the reagent initial vibrational and rotational state restricted to $v=0-3$ and $j=0$ over the collision energy range $0-2.0 \mathrm{eV}$. In the present work, the collision energy and reagent vibrational excitation evolution of the reaction probability defined as $P^{J=0}\left(E_{\mathrm{col}}, v\right)=$ $N_{\mathrm{R}} / N_{\mathrm{T}}$ ( $N_{\mathrm{R}}$ denotes the number of reactive trajectories within the total calculated, $N_{\mathrm{T}}$ ), was calculated with sampled collision energies. For each condition, a batch of 20000 trajectories was necessarily run to obtain results which are converged within $1 \%$ or less. This number increases until $10^{5}$ to achieve enough accurate state specific dynamics. The integration time step in the trajectories is selected to be $0.1 \mathrm{fs}$, a value that guarantees the conservation of both the total energy and total angular momentum. The trajectory was started and finished at a distance between the attacking atom $\mathrm{H}$ and the center of the diatomic molecule $\mathrm{HBr}, R=20 \AA$, far enough to guarantee that the interaction between them is negligible. For non-zero total angular momentum, the appropriate value of the impact parameter, $b_{\max }$, was selected after running preliminarily batches of 10000 trajectories for each collision energy at a fixed value of $b$, systematically increasing the size of $b$ until no reactive trajectories were observed in the 10000 trajectories. For the given collision energy and vibrational state of reagent $\mathrm{HBr}$, ICS is calculated using the expression, $\sigma_{R}=\pi b_{\max }^{2} N_{R} / N_{T}$. The sampling error is defined as $\sigma=\sqrt{\left(N_{T}-N_{R}\right) / N_{T} N_{R}} \times 100 \%$, and the statistical uncertainty of ICS as $\sigma_{r s}=\sigma \sigma_{R}$. As known, a shortcoming of QCT method is its inability to treat the zeropoint energy. In our calculations, we adopt a passive method which simply discards all reactive trajectories in which the final quantum number was smaller than zero (QCT-QMT). ${ }^{47}$

In the present work, we carry out all the calculations on the MB3 PES, which was constructed by modifying the MB2 PES of $1^{2} \mathrm{~A}^{\prime}$ state with spin-orbit (SO) splitting. This PES keeps the main characters of MB2 potential, while it modifies the artificial well in T-shape geometry of $\mathrm{Br}+\mathrm{H}_{2}$ channel. The agreement between experimental rate constants and theoretical ones, calculated using improved transition state theory (TST) with the least-action adiabatic ground-state (LAG) approximation, was much improved on this PES. Figure 1 


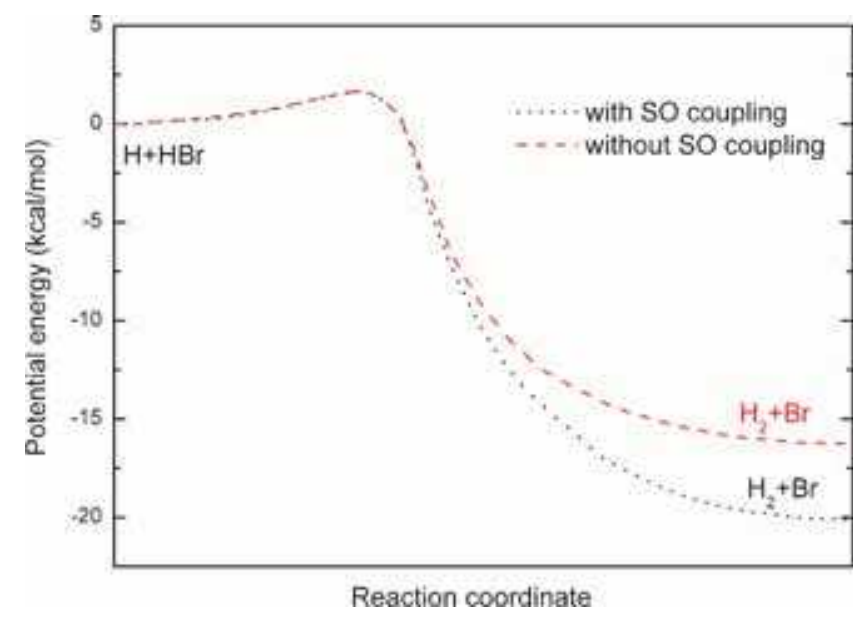

Figure 1. Mininum energy pathways for the collinear configuration. Reactants ( $\mathrm{H}$ and $\mathrm{HBr}$ ) are shown on the left, and product $(\mathrm{H}$ and $\mathrm{HBr})$ are shown on the right. Dotted line represents the potential curve with SO coupling and dashed line without SO coupling.

presents the minimum energy pathways (MEPs) with and without $\mathrm{SO}$ coupling for the $\mathrm{H}+\mathrm{HBr} \rightarrow \mathrm{H}_{2}+\mathrm{Br}$ reaction for the collinear configuration, in which the energy of the reagent asymptote is set as zero. Note that the barrier height for $\mathrm{H}+\mathrm{HBr}$ abstraction is almost not affected by the SO coupling effect. The reaction proceeds with about $20 \mathrm{kcal} / \mathrm{mol}$ exothermicity while considering the SO coupling, which is slightly larger than that without the SO coupling. On the PES, the early barrier height for the collinear approach of $\mathrm{H}$ to molecular $\mathrm{HBr}$ is 1.53 and $21.12 \mathrm{kcal} / \mathrm{mol}$ for perpendicular $\mathrm{H}$ geometries, and no well has been found at T-shaped configuration. More details of the fitting procedure on the potential energy surface can be found in Ref. ${ }^{25,32}$.

\section{Results and Discussion}

In figure 2, we present the current QCT calculated $J=0$ reaction probabilities as a function of collision energy, $E_{\text {col }}$, for $\mathrm{H}+\mathrm{HBr} \rightarrow \mathrm{H}_{2}+\mathrm{Br}$, comparing with the previous QM results obtained by Fu et al..$^{25}$ on the MB3 PES and Xie et al. $^{32}$ on their own PES, respectively. Extensive tests were performed to ensure the proper convergence of the reaction probability. Considering the statistical error, present QCT probabilities are in good agreement with $\mathrm{QM}^{25}$ results. Another feature can be easily captured that the reaction probability quickly arrives at its maximum and then drops smoothly with increasing collision energy. The same phenomenon was also found in the $\mathrm{H}+\mathrm{HCl}$ abstraction. ${ }^{48}$ As pointed out above, the barrier height of abstraction is so small, only 1.53 $\mathrm{kcal} / \mathrm{mol}$ at collinear geometry, that the curves show almost no energy threshold. However, two QM results

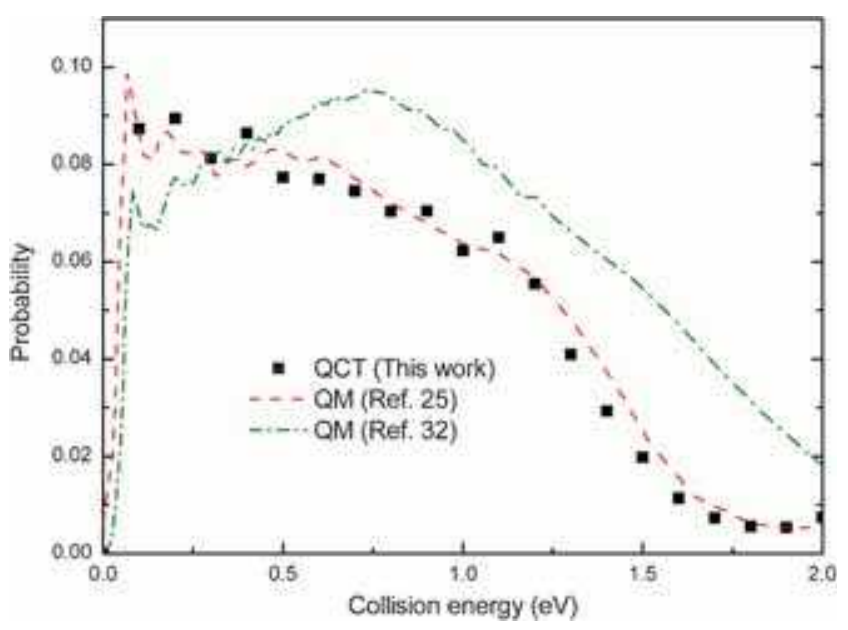

Figure 2. Total reaction probability at $J=0$ as a function of collision energy for the reaction, $\mathrm{H}+\operatorname{HBr}(v=0, j=0)$ $\rightarrow \mathrm{H}_{2}+\mathrm{Br}$. The present QCT results are compared with QM result of Refs. ${ }^{25,32}$

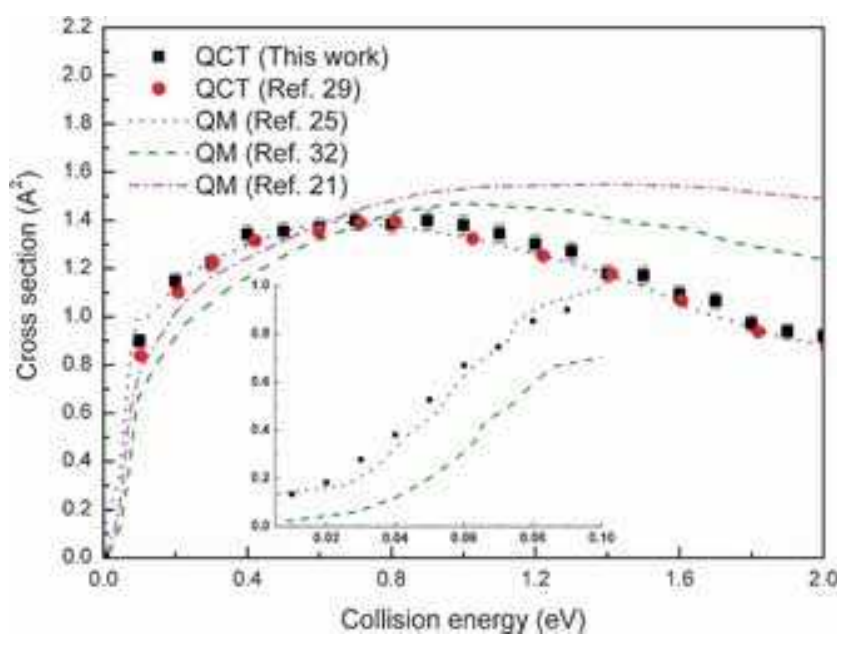

Figure 3. Integral cross sections for the $\mathrm{H}+\operatorname{HBr}(v=0$, $j=0) \rightarrow \mathrm{H}_{2}+\mathrm{Br}$ in the energy range covered in this work. Also included are the QCT results from Ref. ${ }^{29}$ and QM results from Refs. ${ }^{21,25,32}$.

are somewhat different, in particular, from the collision energy corresponding to the maximum probability, apparently due to the different PESs employed in their calculations.

The ICS as a function of the collision energy at initial state $(v=j=0)$ is shown in figure 3 . These results include error bars associated with the statistical uncertainty in the QCT calculation. A key question of the QCT method is its inability to well deal with quantum effects, only the QM calculations, and they can't be neglected in some reactions. To verify our calculations, on the one hand, we compare our QCT cross sections with available theoretical results ${ }^{25,29}$ on MB3 surface. The good agreement between them may suggest that quantum effects, such as zero point energy or 
tunneling, are either negligible or the errors introduced by the quasi-classical treatment cancel each other. On the other hand, to detect the quantum effects in low collision energy region where they could be easily observed, we calculated the ICS by QCT method comparing with QM results ${ }^{25,32}$ in the inner panel of figure 3. As shown, the shape of present work is consistent with the QM results ${ }^{25}$ on the whole, suggesting that the influence of quantum effects on this reaction is not obvious.

Because of the small barrier, the energy threshold for the reaction is very low. Employing the same MB3 $\mathrm{PES}$, the extremely good agreement between $\mathrm{QM}^{25}$ and present QCT results is reasonable. It undergoes a sharp increase below $0.15 \mathrm{eV}$, subsequently a gradual rise to the peak value, $1.39 \AA^{2}$, and then decreases monotonically between 0.7 and $2.0 \mathrm{eV}$. This behavior is well consistent with properties of exothermic reaction processing with about $20 \mathrm{kcal} / \mathrm{mol}$. As a result of characteristics on the different surfaces, the three QM results ${ }^{21,25,32}$ represent the discrepancy to some extent. Aker and coworkers $^{7,8}$ have measured the ICSs of $\mathrm{H}+\mathrm{HX}(\mathrm{X}=\mathrm{Cl}$, $\mathrm{Br}$, I) $\mathrm{H}$ atom abstraction at $E_{\mathrm{col}}=1.6 \mathrm{eV}$, probed by CARS spectroscopy. For $\mathrm{X}=\mathrm{Cl}, \mathrm{Br}$ and $\mathrm{I}$, the experimental ICSs are $2 \pm 1 \AA^{2}, 3 \pm 1 \AA^{2}$ and $2 \pm 1 \AA^{2}$, respectively. However, the uncertainty in the measurement reached the surprising extent, $\pm 33 \%$-50\%. In addition, later experiment ${ }^{49}$ pointed out that the ICS for $\mathrm{H}+\mathrm{HCl}$ should be lower at a special collision energy of $1.6 \mathrm{eV}$. Because of this, we infer that the ICS for the title reaction may be inferior to $2 \AA^{2}$ leading to an approach to the present QCT value, $1.12 \AA^{2}$. From the figure, all theoretical results at $1.6 \mathrm{eV}$ are smaller than the experimental value $3 \pm 1 \AA^{2}$. Hence, further experiments and $\mathrm{PES}$ for $\mathrm{H}+\mathrm{HBr}$ reaction are necessary for the benefit of more accurate ICS.

For atom-diatom reactions (i.e., $\mathrm{A}+\mathrm{BC}$ ), reagent vibrational excitation has been of longstanding interest in reaction dynamics, thus, a focus of present work has been on whether significant influence of vibrational excitation on this reaction can be observed. Initial vibrational state $(v=0-3, j=0)$ selected ICS is depicted in figure 4. As clearly shown in the firgure, strong dependence of ICS on vibrational excitation is observed in the energy region considered here. Note that initial vibrational excitation of $\mathrm{HBr}$ enhances the collision process over the collision energy range. At highstretch excited-states capture-type behavior is observed; i.e., with decreasing collision energy the ICS diverges. Comparing with $v=0$ results at low collision energies, the ICSs show unusually large values at three $\mathrm{HBr}$ quantum numbers. The values for $v=1,2$ and 3 are 2.7, 4.6 and 4.8 at $0.1 \mathrm{eV}$ collision energy respectively. This can

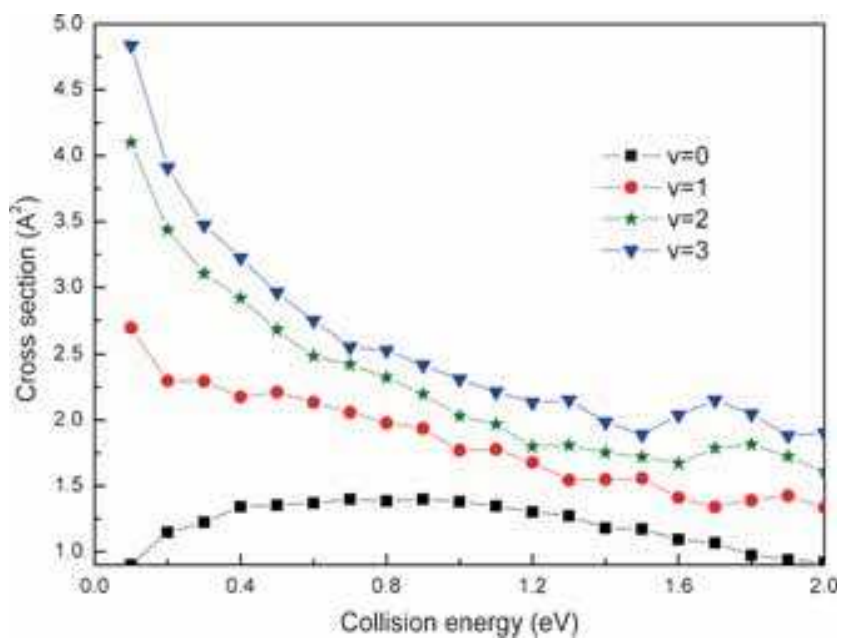

Figure 4. Integral cross sections of $\mathrm{H}+\mathrm{HBr}(v=0,1,2,3$, $j=0) \rightarrow \mathrm{H}_{2}+\mathrm{Br}$ as a function of collision energy.

be attributed to the extension of the impact parameter domain that allows reaction and leads to larger ICSs, as the opacity functions at different collision energies shown in figure 8(a). Clearly, the capture-type dynamics arises with vibrational quantum number $v$, because at large separations the potential influencing the motion of the attacking $\mathrm{H}$ atom is attractive.

The dynamic behavior of vibrationally excited $\mathrm{HBr}$ discussed above is beyond the applicability of Polanyi's rules. ${ }^{50,51}$ Actually, extreme vibrational excitation of reagent has been amply discussed for $\mathrm{H}+\mathrm{H}_{2} \mathrm{O}^{52}$ and $\mathrm{H}+\mathrm{HF}^{53}$ abstraction reactions. For the title reaction, as soon as the vibrational energy in the $\mathrm{HBr}$ molecule exceeds the barrier $(1.53 \mathrm{kcal} / \mathrm{mol})$ at $v=1$, the excitation function could easily switch from activated at $v=0$ to capture-type beginning with $v=1$ shown in figure 4. For the L-L-H ${ }^{54}$ ( $\mathrm{L}$ and $\mathrm{H}$ denote light and heavy) mass combination reaction system, the reaction dynamics are to be kinetic control. Thus vibrational energy in $\mathrm{HBr}$ molecule can be converted into kinetic energy helping overcome the barrier. The switch can be induced by the interaction between the attacking atom and the vibrating molecule. When the $\mathrm{HBr}$ molecule is highly excited, the large energy of the system makes the attacker observe an almost free $\mathrm{H}$ atom. As the internal energy is very high, passing the small barrier is actually a downhill motion on the PES. Just like an attractive system, the slower the approach, the larger the chance that the attacker is captured and reacts. We also found that the influence of reagent vibrational excitation on ICS seems to be more marked at low collision energy up to $0.5 \mathrm{eV}$. For instance, the ratio of ICS $\sigma(v=3) / \sigma(v=1)$ at $0.1 \mathrm{eV}$ is equal to 1.81 , with the corresponding ratio value of 1.41 at $2.0 \mathrm{eV}$. 

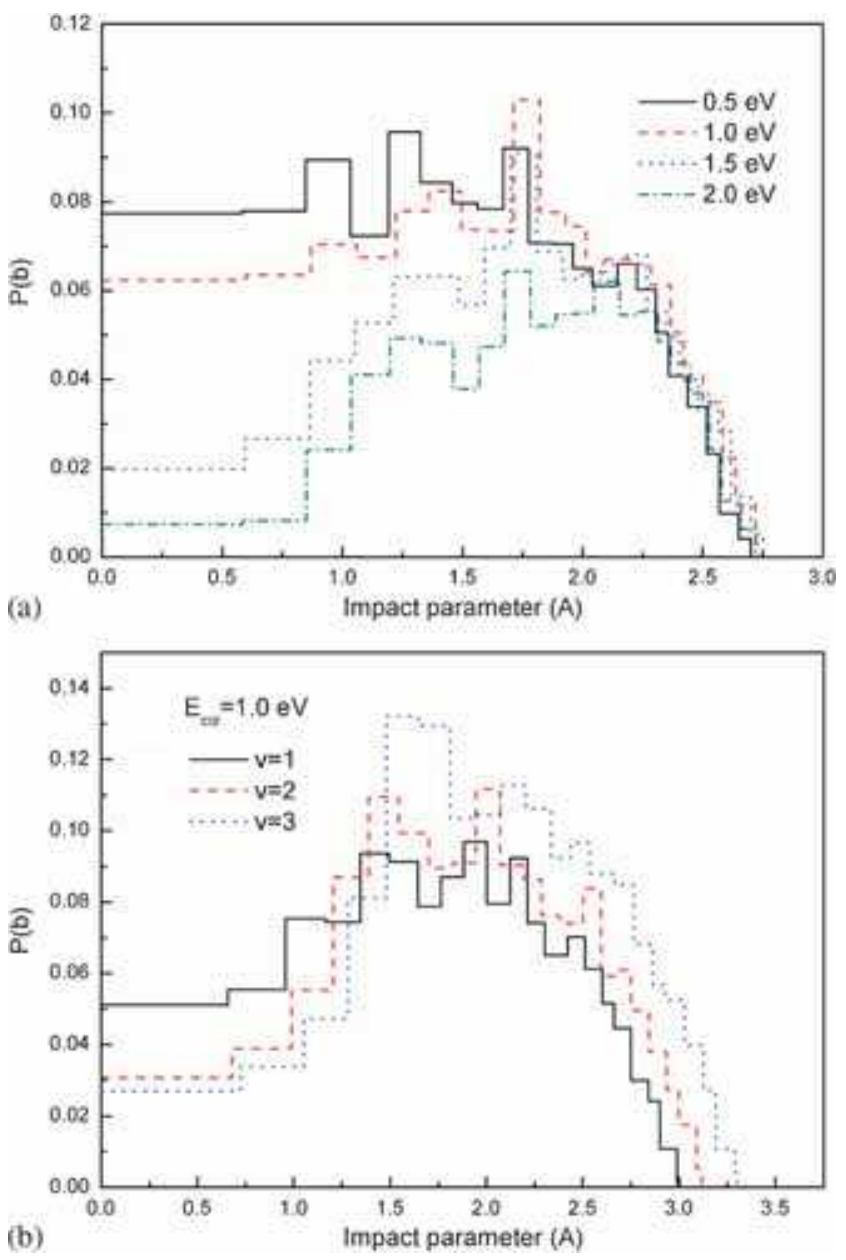

Figure 5. Opactiy functions for the $\mathrm{H}+\operatorname{HBr}(v, j=0)$ $\rightarrow \mathrm{H}_{2}+\mathrm{Br}$ (a) at collision energies of $0.5,1.0,1.5,2.0 \mathrm{eV}$ with $v=0$ and (b) at $1.0 \mathrm{eV}$ collision energy with $v=1-3$.

Observing figure 5, where we plot the opacity function, the reactivity is very sensitive to the collision energy and the vibrational excitation of the reagent $\mathrm{HBr}$ molecule. If the reagent is vibrationally unexcited, the maximum impact parameter where reactive collisions are observed does not exceed $3 \AA$. When $b=0$, shown in the upper panel, $\mathrm{P}(b)$ representing the reaction probability, decreases with collision energy, which is consistent with the behavior of figure 2 . We can see that the reactivity regularly increases and then drops along with the different impact parameters, $b$. At higher collision energies of 1.5 and $2.0 \mathrm{eV}$, a bell shape is found with peak at about $b=1.75 \AA$. It suggests that the collision with small impact parameter is blocked at high collision energies. Obviously, collisions with larger $b$ become significant and no difference is observed at the largest impact parameter bin. For certifying the results of figure 4, we also plot the reaction probability for $v=1$, 2,3 and $j=0$ in the bottom panel at a collision energy of $1.0 \mathrm{eV}$. Clearly, the reaction ends with larger

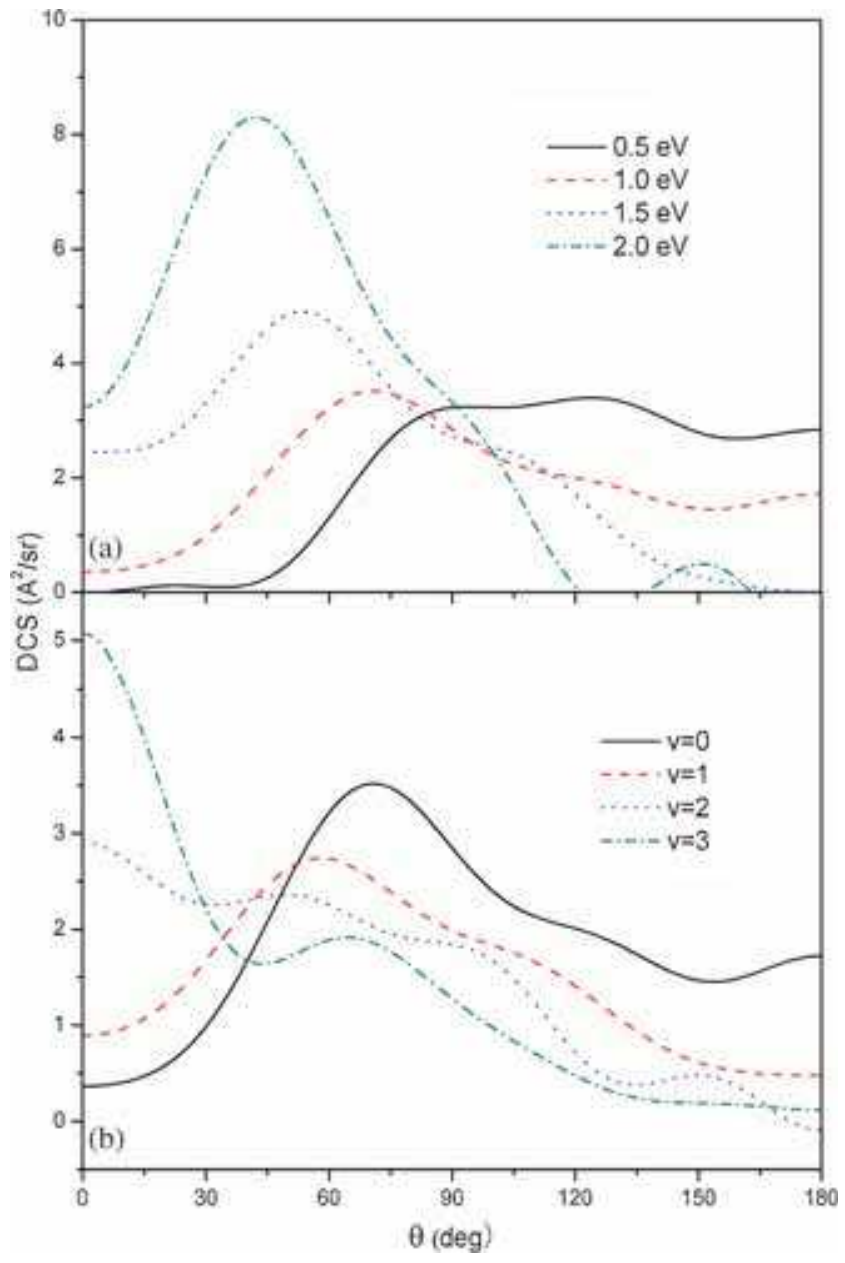

Figure 6. Total reactive differential cross sections (DCSs) for (a) different collision energies and (b) $\mathrm{HBr}$ vibrational excitations at a collision energy of $1.0 \mathrm{eV}$.

$b$ as vibrational quantum number varies from 1 to 3 , resulting in the increase of ICS indicated in figure 4.

So far, we have focused on analyzing reactivity over the collision energy and reagent vibrational quantum number range. Considering the direction in which the products are scattered could help to reveal more information on reaction mechanisms. Therefore, differential cross sections (DCSs) have been calculated for the four discrete collision energies, $0.5,1.0,1.5$ and 2.0 $\mathrm{eV}$ in figure 6(a), and for $v=0-3$ and $j=0$ levels of reagent $\mathrm{HBr}$ at a fixed collision energy of $1.0 \mathrm{eV}$ in figure 6(b). In the first case, the DCSs strongly associate with energy and peak in the forward hemisphere in the most cases except for the lowest energy $E_{\mathrm{col}}=0.5 \mathrm{eV}$. Along with the L-L-H model, it is reasonable that for higher collision energies the product would prefer a forward scatter on the attractive PES. For 0.5 and $1.0 \mathrm{eV}$, the $\mathrm{H}_{2}$ are scattered broadly backward-sideways, indicating a direct rebound-type mechanism. ${ }^{14,33}$ Namely, both the new bond $\mathrm{H}-\mathrm{H}$ forming and the old bond $\mathrm{H}-\mathrm{Br}$ 
break occur at the same time. Clearly, the direct mechanism is the result of collinear approach of reagents in low energy regime. To clearly show this case, we have observed lots of reactive trajectories and plot a typical trajectory at $E_{\text {col }}=2.0 \mathrm{eV}$ in figure 7(a). As can be seen, the trajectory displayed a very direct reaction as emphasized above. Importantly, indirect microscopic mechanism was also found in the abstraction by counting the turning points on reactive trajectories. ${ }^{29,33}$ Figure 7(b) presents the evolution of the three inter-nuclear distances over time of another trajectory at $E_{\text {col }}=0.2$ $\mathrm{eV}$. This phenomenon of one reaction involving two different mechanisms was also observed in $\mathrm{H}+\mathrm{HS}$ abstraction $^{55}$ and $\mathrm{F}+\mathrm{HCl} \rightarrow \mathrm{HF}+\mathrm{Cl}^{56}$ In the panel (b), the $\mathrm{Br}-\mathrm{H}$ distance reaches its lowest value before the $\mathrm{H}$ $\mathrm{H}$ does. In other words, before forming the product $\mathrm{H}_{2}$, the incoming atom $\mathrm{H}$ can first hit more than once the $\mathrm{Br}$ end of $\mathrm{HBr}$ molecule from any random angles, and then migrates to the $\mathrm{H}$ end and inserts to form the $\mathrm{H}_{2}$ product. Valentini ${ }^{57}$ has pointed out that the direct reaction mostly takes place follow the collinear minimum energy path at high collision energies, except the overshoots and collision with repulsive inner wall. In this reaction, clearly, trajectory (first panel) evolves close to the MEP and direct mechanism plays a predominate role. For higher collision energies 1.5 and $2.0 \mathrm{eV}$, however, the angle of scattering preference of the molecular product shifts towards the forward direction, which is consistent with previous reports. ${ }^{29,32}$ For the title reaction, the reaction coordinate before the early barrier is characterized mainly by the motion of attacking hydrogen atom. With increasing the collision energy, the substantial momentum of attacking hydrogen atom promotes the motion of product $\mathrm{H}_{2}$ along the continuation of the direction of the attacking atom. Additionally, we can see that the scattering peaks higher and scattering shifts towards a smaller angle range, this behavior of scattering degree increasing with collision energy may contribute to the impact effect.

To get a better idea of DCS at different vibrational states, we have depicted the DCSs at $v=0-3$ and $j=0$ levels of reagent $\mathrm{HBr}$ at a fixed collision energy of 1.0 $\mathrm{eV}$ in figure 6(b). As shown, the influence of reagent vibrational excitation on DCS is significant. Similar to the collision energy, it varies the product $\mathrm{H}_{2}$ scattering from sideways, as it seems, to absolutely forward direction. For $v=0,1$, the peaks locate in scattering angle range $50-90^{\circ}$, while for $v=2,3$, the scattering peaks in the forward direction $\left(\theta=0^{\circ}\right)$. It can also be seen that the forward scattering intensity increases with higher reagent vibrational excitation levels. As already noted, the vibrational energy above the barrier could be converted into kinetic energy to the benefit of the direct mechanism.

The QCT method is limited by the fact that the calculated product vibrational and rotational quantum numbers are actually not integer. The usual histogram binning (HB) method to assign the quantum number $v^{\prime}$ and $j^{\prime}$ of the diatomic $\mathrm{H}_{2}$ product consists in rounding the classical actions to their nearest integers. Sometimes, this procedure yields some typical discrepancies between QCT and QM results. To overcome this problem, the Gaussian binning (GB) method ${ }^{58}$ was adopted to determine the population in final states to improve the QCT results in this work. A full width at half maximum of the Gaussian functions was set to be $\Delta=0.1$, which has shown a good agreement between QCT and quantum distributions.

Figure 8 depicts the product rovibrational distributions, that is, the ICS as a function of the rovibrational states of $\mathrm{H}_{2}$ at four specific collision energies marked. The upper panel and lower panel show $\mathrm{H}_{2}$ vibrational
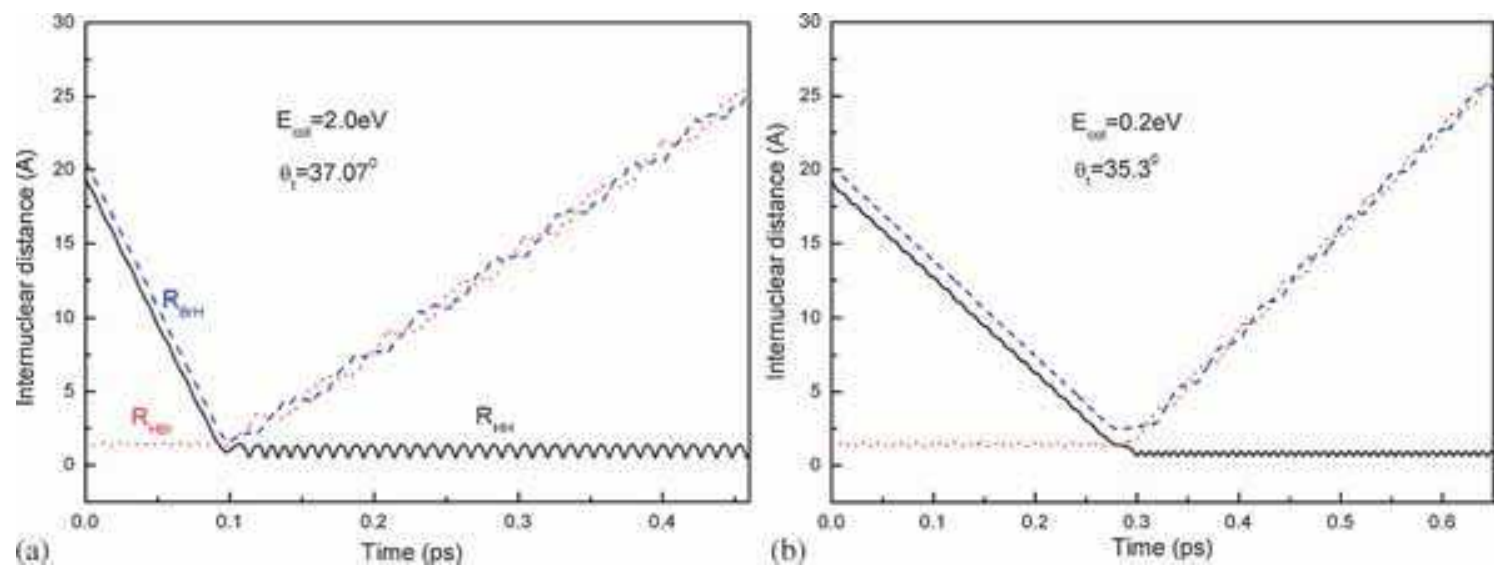

Figure 7. Internuclear distances of $\mathrm{HH}, \mathrm{HBr}$ and $\mathrm{BrH}$ as a function of propagation time with scattering angles of (a) $\theta_{\mathrm{r}}=37.07^{\circ}$ at $E_{\mathrm{col}}=2.0 \mathrm{eV}$ and (b) $\theta_{\mathrm{r}}=35.3^{\circ}$ at $E_{\mathrm{col}}=0.2 \mathrm{eV}$. 

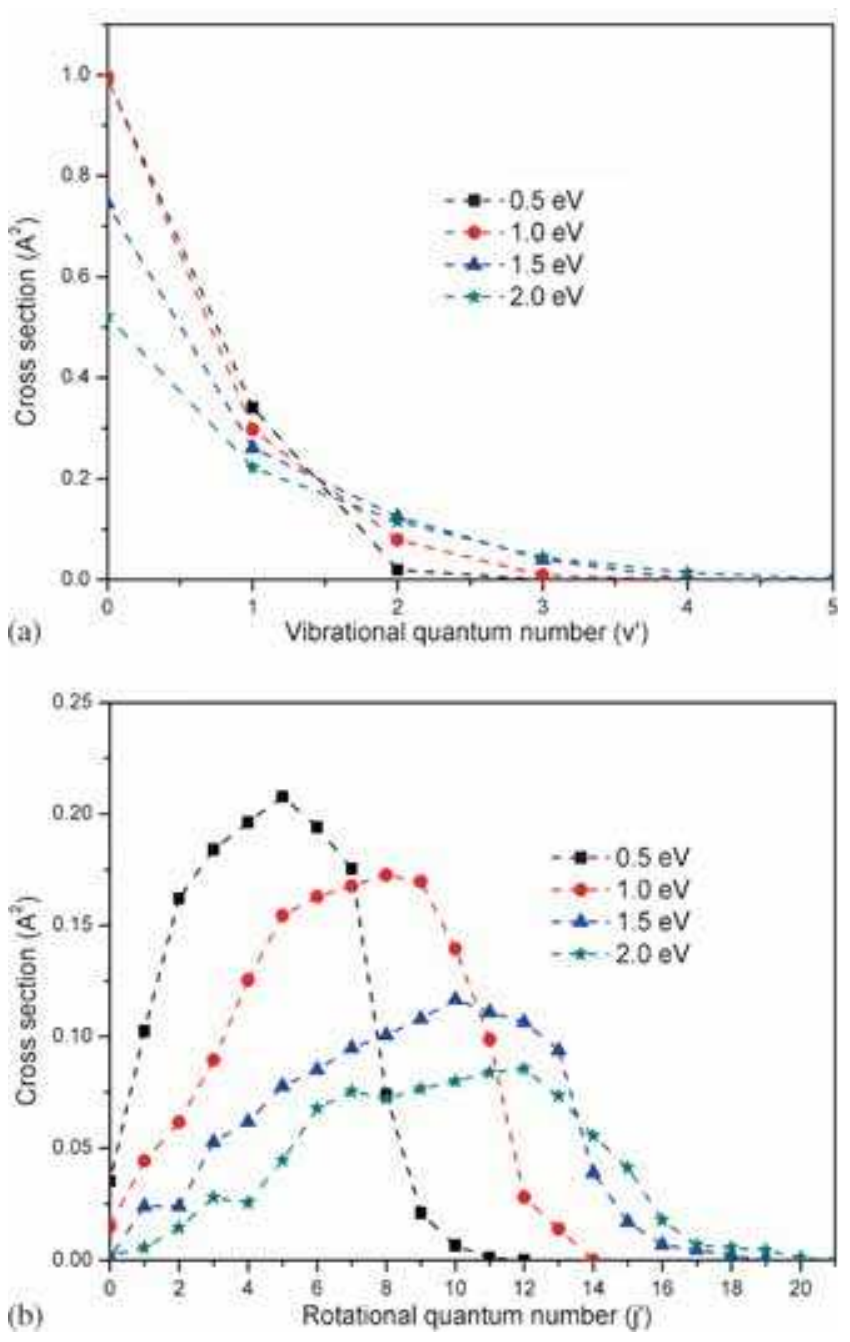

Figure 8. Rovibrational state-resolved cross sections for the $\mathrm{H}+\mathrm{HBr}(v=0, j=0) \rightarrow \mathrm{H}_{2}\left(v^{\prime}, j^{\prime}\right)+\mathrm{Br}$ at four collision energies and final quantum number $v^{\prime}$ and $j^{\prime}$ for (a) the vibrational state distributions and; (b) the rotational state distributions.

and rotational distributions respectively. As seen from the upper panel, the most populated vibrational level is $v^{\prime}=0$, and the $\mathrm{H}_{2}$ products are highly vibrationally excited with $v^{\prime}$ up to its maximum, $v^{\prime}=5$, which suggests the reduction of open rotational states in a given vibrational manifold. Further, the ICSs for the final states $v^{\prime}<2$ decrease with collision energy, whereas increase for product $\mathrm{H}_{2}\left(v^{\prime} \geq 2\right)$, which means that more highly vibrationally hot $\mathrm{H}_{2}$ products produce. From figure 8(b) for rotational distributions of $\mathrm{H}_{2}$ at four collision energies, we can see that each product rotational state distribution presents bell-shaped curves. The distribution peaks decay and shift to higher $j^{\prime}$ values with collision energy, that is, the higher the collision energy increases, the more rotationally excited the product $\mathrm{H}_{2}$ is. Clearly, the rotational distributions appear to be broader with an increase of collision energy.
It is very interesting that the rovibrational stateresolved cross sections for the reaction, $\mathrm{H}+\mathrm{HBr}(v=1$, $j=0) \rightarrow \mathrm{H}_{2}\left(v^{\prime}, j^{\prime}\right)+\mathrm{Br}$ display the same trend comparable with figure 8 . Hence, the results are not shown here.

\section{Conclusion}

In this paper, we have performed a detailed dynamical study of $\mathrm{H}+\mathrm{HBr}(v=0-3, j=0) \rightarrow \mathrm{H}_{2}+\mathrm{Br}$ reaction with QCT method on an accurate global potential energy surface at large $\mathrm{HBr}$ vibrational energies with the purpose of connecting the extremely large vibrational enhancement of the reactivity with the features of potential surface and the mass combination, over a wide range of collision energies.

Both present QCT and available QM results for reaction probability and ICS for the $\mathrm{HBr}$ ground state $(v=0, j=0)$, are found to be fairly consistent with each other on MB3 surface. Moreover, we investigated the influence of reagent vibrational excitation up to $v=3$ on reaction dynamics. An important remark to note in this work is that the reaction switches from activated to capture-type with increase of the quantum number $v$. This divergence of unusually large ICS at low collision energy is attributed to the extension of impact parameter resulting in the large cone of acceptance. On the attractive PES, the system with high internal energy passes the barrier as a downhill motion. For low collision energies, the slower the approach of attacking $\mathrm{H}$ atom, larger is the chance that the attacker is captured. This behavior of highly vibrationally excited reagent enhances the reactivity of the $\mathrm{H}+\mathrm{HBr}$ reaction.

The calculated DCS indicates that the product molecule $\mathrm{H}_{2}$ prefers the forward scattering, and the roles of collision energy and initial $v$ appear to affect the direction of scattering in a similar way. Direct and migration reaction mechanisms were applied to explain this behavior. Further analysis shows that the direct reaction is the predominant process in these two mechanisms.

For the purpose of verifying our results, it is hoped that more accurate theoretical and experimental exploration dealing with the effect of excited $\mathrm{HBr}$ for this reaction will be reported.

\section{Acknowledgements}

We thank Prof. Y. Kurosaki for providing the MB3 potential energy surface, and Prof Ke-Li Han for providing the code. 


\section{References}

1. Husain D and Slater N K H 1980 J. Chem. Soc., Faraday Trans. 76276

2. Jourdain J L, Lebras G and Combourieu J 1981 Chem. Phys. Lett. 78483

3. Umemoto H, Wada Y, Tsunashima S, Takayanagi T and Sato S 1990 Chem. Phys. 143333

4. Seakins P W and Pilling M J 1991 J. Phys. Chem. 95 9878

5. Talukdar R K, Warren R F, Vaghijiani G L and Ravishankara A R 1992 Int. J. Chem. Kinet. 24973

6. Mitchell T J, Gonzalez A C and Benson S W $1995 \mathrm{~J}$. Phys. Chem. 9916960

7. Aker P M, Germann G J and Valentini J J 1989 J. Chem. Phys. 904795

8. Aker P M, Germann G J, Tabor K D and Valentini J J 1989 J. Chem. Phys. 904809

9. Aker P M and Valentini J J 1993 Int. Rev. Phys. Chem. 12363

10. Pomerantz A E, Camden J P, Chiou A S, Ausfelder F, Chawla N, Hase W L and Zare R N 2005 J. Am. Chem. Soc. 12716368

11. Zhang J Y, Jankunas J, Bartlett N C M, Goldberg N T and Zare R N 2010 J. Chem. Phys. 132084301

12. White J M and Thompson D L 1974 J. Chem. Phys. 61 719

13. Sudhakaran M P and Raff L M 1985 Chem. Phys. 95165

14. Aker P M and Valentini J J 1990 Isr. J. Chem. 30157

15. Lynch G C, Truhlar D G, Brown F B and Zhao J G 1995 J. Phys. Chem. 99207

16. Mielke S L, Tawa G J, Truhlar D G and Schwenke D W 1995 Chem. Phys. Lett. 23457

17. Kurosaki Y and Takayanagi T 2003 J. Chem. Phys. 119 7838

18. Kurosaki Y and Takayanagi T 2005 Chem. Phys. Lett. 406121

19. Kurosaki $\mathrm{Y}$ and Takayanagi T private communication

20. Jiang B, Xie C J and Xie D Q 2011 J. Chem. Phys. 134 114301

21. Zhang A J, Zhang P Y, Chu T S, Han K L and He G Z 2012 J. Chem. Phys. 137194305

22. Quan W L, Song Q and Tang B Y 2007 Chem. Phys. Lett. 437165

23. Quan W L, Song Q and Tang B Y 2007 Chem. Phys. Lett. 442228

24. Quan W L, Song Q and Tang B Y 2007 Int. J. Quantum. Chem. 107657

25. Fu B N and Zhang D H 2007 J. Phys. Chem. A 1119516

26. Fu B N, Zhou Y and Zhang D H 2008 J. Theor. Comput. Chem. 7777

27. Takayanagi T and Kurosaki Y 2000 Chem. Phys. Lett. 1137158

28. Panda A N 2008 J. Phys. Chem. A 1125327

29. Zhang W Q, Cong S L, Zhang C H, Xu X S and Chen M D 2009 J. Phys. Chem. A 1134192
30. Panda A N, Herráez-Aguilar D, Jambrina P G, Aldegunde J, Althorp S C and Aoiz F J 2012 Phys. Chem. Chem. Phys. 1413067

31. Liang J J, Yang C L, Wang L Z and Sun Z G 2012 Chem. Phys. 392180

32. Xie C J, Jiang B and Xie D Q 2011 J. Chem. Phys. 134 184303

33. Pomerantz A E, Camden J P, Chiou A S, Ausfelder F, Chawla N, Hase W L and Zare R N 2005 J. Am. Chem. Soc. 12716368

34. Zhang Y C, Zhang J Z H and Kouri D J 1988 Phys. Rev. Lett. 602367

35. Mielke S L, Truhlar D G and Schwenke D W $1995 \mathrm{~J}$. Phys. Chem. 9916210

36. Volobuev Y L, Hack M D and Truhlar D G 1999 J. Phys. Chem. A 1036225

37. Panda A N and Althorpe S C 2007 Chem. Phys. Lett. 43950

38. Xie C J, Jiang B, Xie D Q and Sun Z G 2012 J. Chem. Phys. 136114310

39. Brownsword R A, Kappel C, Schmiechen P, Upadhyaya H P and Volpp H R 1998 Chem. Phys. Lett. 289241

40. Li R J, Han K L, Li F E, Lu R C, He G Z and Lou N Q 1994 Chem. Phys. Lett. 220281

41. Han K L, He G Z and Lou N Q 1996 J. Chem. Phys. 105 8699

42. Wang M L, Han K L and He G Z 1998 J. Phys. Chem. A 10210204

43. Zhang L, Chen M D, Wang M L and Han K L $2000 \mathrm{~J}$. Chem. Phys. 1123710

44. Han K L, Xu D L, He G Z and Lou N Q 2001 J. Phys. Chem. A 1052956

45. Chen M D, Han K L and Lou N Q 2002 J. Chem. Phys. 283463

46. Chen M D, Han K L and Lou N Q 2003 J. Chem. Phys. 1184463

47. Truhlar D G 1979 J. Phys. Chem. 83188

48. Brownsword R A, Kappel C, Schmiechen P, Upadhyaya H P and Volpp H R 1998 Chem. Phys. Lett. 289241

49. Barclay V J, Collings B A, Polanyi J C and Wang J H 1991 J. Phys. Chem. 952921

50. Polanyi J C 1972 Acc. Chem. Res. 5161

51. Polanyi J C 1987 Science 236680

52. Schatz G C, Wu G, Lendvay G, Fang D C and Harding L B 1999 Faraday Discuss. 113151

53. Bene E, Lendvay G and Pta G 2005 J. Phys. Chem. A 1098336

54. Wang M L, Han K L and He G Z 1998 J. Chem. Phys. 1095446

55. Duan Z X, Li W L, Xu W W and Lv S J 2013 J. Chem. Phys. 139094307

56. Duan Z X, Li W L and Qiu M H 2012 J. Chem. Phys. 136144309

57. Picconatto C A, Srivastava A and Valentini J J $2001 \mathrm{~J}$. Chem. Phys. 1141663

58. Bonnet L and Rayez J C 2004 Chem. Phys. Lett. 397106 